\title{
COMMUNICATION
}

\section{Functional nano-structuring of thin silicon nitride membranes}

\author{
Milan Matějka*, Stanislav Krátký, Tomáš Řiháček \\ Alexandr Knápek, Vladimír Kolařík ${ }^{1}$
}

\begin{abstract}
The paper describes the development and production of a nano-optical device consisting of a nano-perforated layer of silicon nitride stretched in a single-crystal silicon frame using electron beam lithography (EBL) and reactive ion etching (RIE) techniques. Procedures for transferring nanostructures to the nitride layer are described, starting with the preparation of a metallic mask layer by physical vapor deposition (PVD), high-resolution pattern recording technique using EBL and the transfer of the motif into the functional layer using the RIE technique. Theoretical aspects are summarized including technological issues, achieved results and application potential of patterned silicon nitride membranes.
\end{abstract}

K e ywords: membrane, nano optical device, electron optics, electron beam lithography, silicon nitride, reactive ion etching, silicon etching, microfabrication

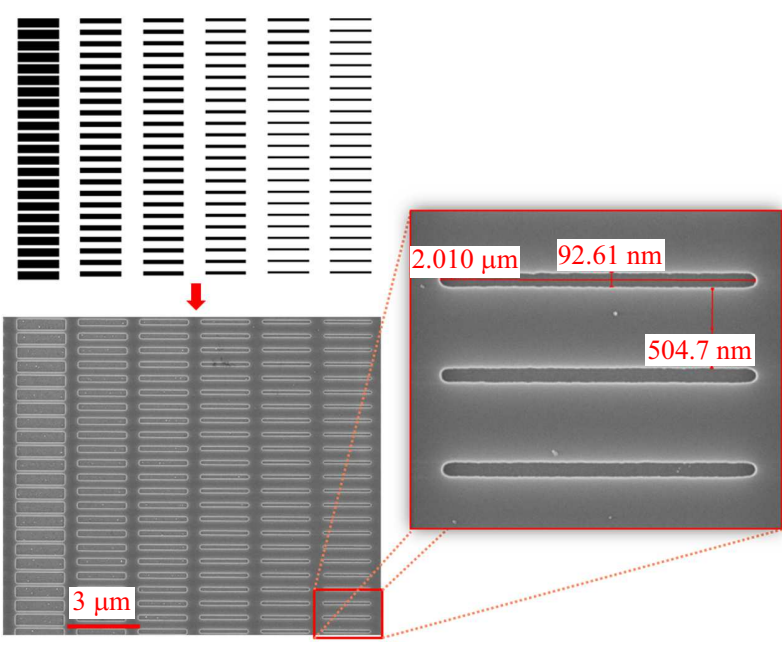

Fig. 1. Pictures of test design with different pattern sizes and SEM images of the pattern after RIE of silicon nitride

\section{Introduction}

Silicon nitride (SiN) layers are typically used in semiconductor industry and IC industry as insulating or masking layers in MOS technology [1]. SiN films are commonly used as well as a base material for membrane fabrication that can be used in various microelectronic and micro optic devices (MEMS, MOMS), eg as a capacitive pressure sensor with a $\mathrm{Si}_{3} \mathrm{~N}_{4}$ diaphragm [2].

The basic procedure of membrane fabrication is well known [3][4]. Typically, it is obtained by wet anisotropic etching of bulk monocrystalline (100) silicone coated with a silicon nitride layer. What brings new technological challenges is the development of the fabrication process, which deals with the above-mentioned IC functional micro- and nanopatterning of the membrane surface. Such innovative membrane-based devices can have both new functionality and possible applications, eg, modification of electromagnetic radiation or of the charged particle beams distribution, phase or spectrum. Recently, a series of research activities and publications have been devoted to the development of these functional elements, eg, micro-optical elements for Helium [5], electron microscopy [6], laser and light optic [7][8], as well as nanosieves for molecular separation in microfluidics or for bioengineering [9].

In this article, we propose a fabrication method of a patterned membrane based Nano Optical Device (NOD) with application in charged particle optics. The membrane is not used just as a support for functional structures built on top (not IC based), but they are used as a bulk material to prepare functional relief structures directly in the SiN layer itself. To do so, different etching techniques have to be adapted within the fabrication steps: plasmatic etching $(\mathrm{PE})$, reactive-ion etching (RIE) and anisotropic wet silicon etching. High-resolution e-beam lithography (EBL) is necessary due to the resolution required for patterning of planar structures in the membrane.

\section{Design and fabrication of the test structures}

The design of test structure consists of a series of lines on an area of approximately $20 \times 20 \mu \mathrm{m}^{2}$ (Fig. 1).

\footnotetext{
1 Institute of Scientific Instruments of the CAS, v. v. i., Královopolská 147, 61264 Brno, Czech Republic, * Corresponding author: milan.matejka@isibrno.cz
}

DOI: $10.2478 /$ jee-2020-0019, Print (till 2015) ISSN 1335-3632, On-line ISSN 1339-309X

(C) This is an open access article licensed under the Creative Commons Attribution-NonCommercial-NoDerivs License (http://creativecommons.org/licenses/by-nc-nd/3.0/). 


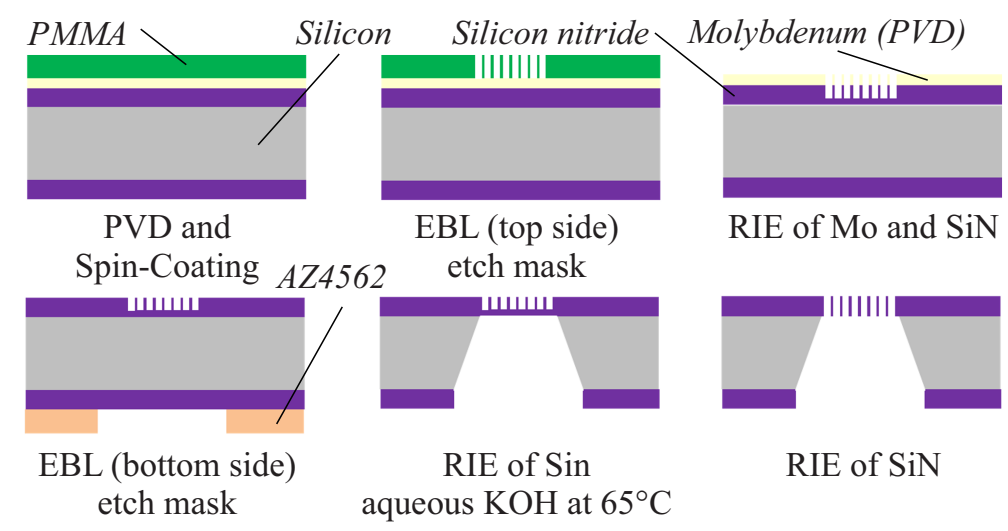

Fig. 2. Schematic of fabrication processes of silicon nitride patterned membranes.the thickness of silicon nitride is 100 nm, the thickness of molybdenum is $20 \mathrm{~nm}$. with extra RIE etching at the end of the process to finish holey pattern in the membrane, the final thickness of the silicon nitride membrane is $75 \mathrm{~nm}$

We used it to recognize the parameters of motif transfer in individual fabrication steps: EBL soft mask, RIE hard mask and RIE SiN. The parameters of the lines in the test structure were as follows: line width 50, 70, 100, 150, 200 and $400 \mathrm{~nm}$, line length $2000 \mathrm{~nm}$, line periodicity $600 \mathrm{~nm}$.

The sample with the test structure was prepared from a monocrystalline silicon wafer (100) with $100 \mathrm{~nm}$ of silicon nitride (LPCVD low-tension) on both sides and 20 $\mathrm{nm}$ of molybdenum (Mo) prepared by PVD. For etching of Mo and SiN, we used an RIE unit with inductively coupled plasma (ICP) of Oxford Instruments. This test structure was measured and evaluated using an atomic force microscope (AFM) and an electron microscope (SEM) between the etching steps to estimate how the line motif would expand during hard mask preparation and then during motif transfer across the hard mask into the nitride layer.

The critical part was to find correct parameters of RIE for motif transfer with low edge extension, high selectivity, and low etching speed.

With respect to our previous experience [10][11], we have chosen the $\mathrm{SF}_{6}+\mathrm{Ar}$ and $\mathrm{CF}_{4}+\mathrm{CHF}_{3}$ recipes for molybdenum mask and silicon nitride etching. From the measurements of the sample with the test structure in SEM, AFM and profilometry we were able to estimate good starting parameters for the RIE process. For the Mo hard mask preparation we used a recipe with $\mathrm{SF}_{6}$ $(50 \mathrm{sccm})+\operatorname{Ar}(40 \mathrm{sccm})$ gas mixure, ICP power 3000 $\mathrm{W}$ and $10 \mathrm{~W}$ RIE power at $60^{\circ} \mathrm{C}$. This recipe exhibits an average etching rate of molybdenum $2 \mathrm{~nm} / \mathrm{s}$. Although the selectivity of Mo/PMMA etching is not high, it is sufficient for the purpose. For the silicon nitride etching through the hard mask we used a recipe with $\mathrm{CF}_{4}$ (10 $\mathrm{sccm})+\mathrm{CHF}_{3}(40 \mathrm{sccm})$ gases, ICP power $1000 \mathrm{~W}, 50$ $\mathrm{W}$ RIE power and working temperature $60^{\circ} \mathrm{C}$. This recipe exhibits an average SiN etching rate of $2.5 \mathrm{~nm} / \mathrm{s}$ and at the same time, the hard mask (Mo) etch rate is about 20 times lower.

\section{Fabrication procedure characterization}

After initial study with the test structure, we have proposed the technological process of fabrication of a real nano-structured membrane. The fabrication processes are schematically illustrated in Fig. 2. and are as follows:

Top-side lithography operations

- PVD deposition of Mo layer (hard mask)

- coating of the PMMA resist

- pattern recording by EBL at EBPG5000+ES (soft mask)

- plasmatic etching of the PMMA in $\mathrm{O}_{2}$

- RIE of the Mo layer trough the PMMA mask

- RIE of the SiN layer trough the Mo mask

- plasmatic ashing of PMMA in $\mathrm{O}_{2}$ (dry removal of PMMA)

- wet removal of the Mo layer $\left(\mathrm{H}_{3} \mathrm{PO}_{4}: \mathrm{HNO}_{3}: \mathrm{H} 2 \mathrm{O}\right)$.

- protective coating with AZ4562 resist Back-side lithography operations

- coating of the AZ4562 resist on back-side

- pattern recording by EBL at EBPG5000+ES (soft mask)

- RIE of the SiN layer trough the AZ4562 mask

- wet removal resist

- wet etching of silicon in aqueous $\mathrm{KOH}$ solution at $65^{\circ}$ CFinalization

- RIE of the SiN layer without mask

In order to ensure the correct recording of the structure into the membrane, a number of measurement operations by AFM, SEM and a probe profilometery have to be included. These measurements take place especially between individual etching steps by RIE and during the mask preparation steps.

\section{Design of the membrane based nano-optical device}

The design of the nano optical device (NOD is inspired by the concept published in [12]. The NOD we have de- 


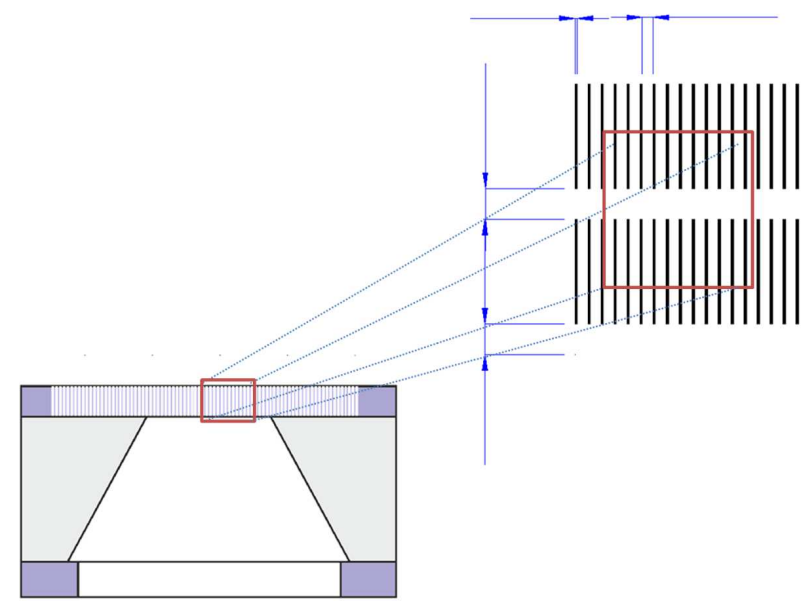

Fig. 3. Schematic illustration of NOD silicon chip with holey grating pattern transferred directly in the nitride membrane.grating parameters: linewidth $70 \mathrm{~nm}$, periodicity $250 \mathrm{~nm}$, length $2000 \mathrm{~nm}$

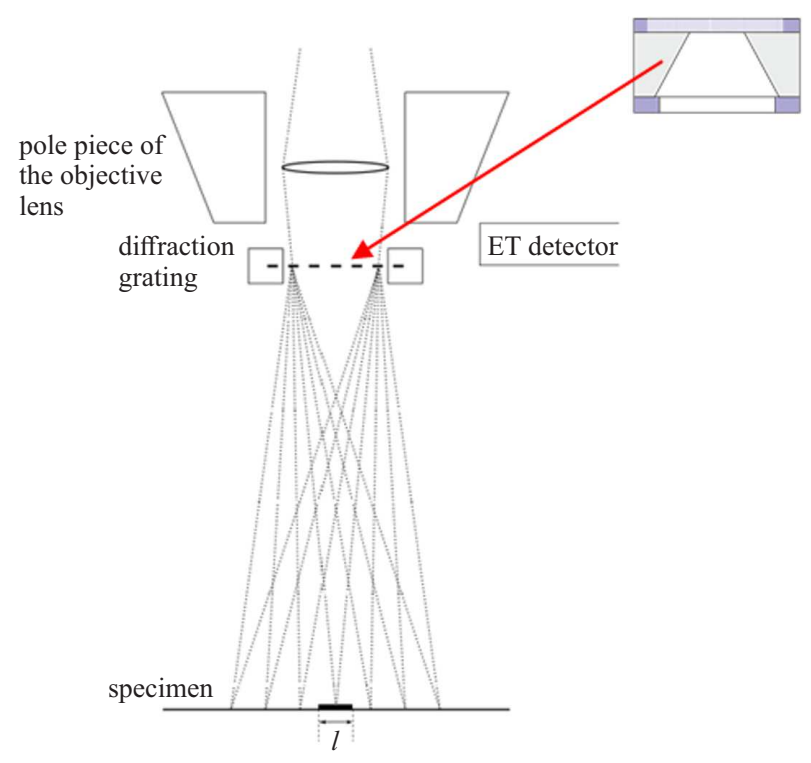

Fig. 4. Scheme of the experiment with membrane base NOD in the SEM magellan 400

signed (see Fig. 3) purposefully for experimental study of electron beam diffraction in a scanning electron microscope. The device was developed as part of research into the possibility of phase manipulation in the primary electron beam in transmission or scanning electron microscopes.

One of the most promising areas in which this research is directed is the possibility of generating vortex beams. The application potential is, for example, probing of magnetic materials or for electron spin-polarized beam microscopy [13].

To fabricate the designed NOD, we used the technology we have developed and described above in this paper. The NOD is a silicon chip with external dimensions not exceeding $44 \mathrm{~mm}^{2}$. In the middle of the chip there is a $100 \times 100 \mu \mathrm{m}^{2}$ window with a $70 \mathrm{~nm}$ thick ni- tride membrane. The membrane area and the area around $(1 \times 1 \mathrm{~mm} 2)$ contains high-density rectangular shape nano-perforation with a size of $70 \mathrm{~nm} \times 2000 \mathrm{~nm}$, see Fig. 5.

The membrane surface is metalized with a thin layer of platinum to avoid charging. This device functions in the electron microscope as a screen with over $20 \mathrm{k}$ nano apertures on which diffraction occurs of the passing electron beam.

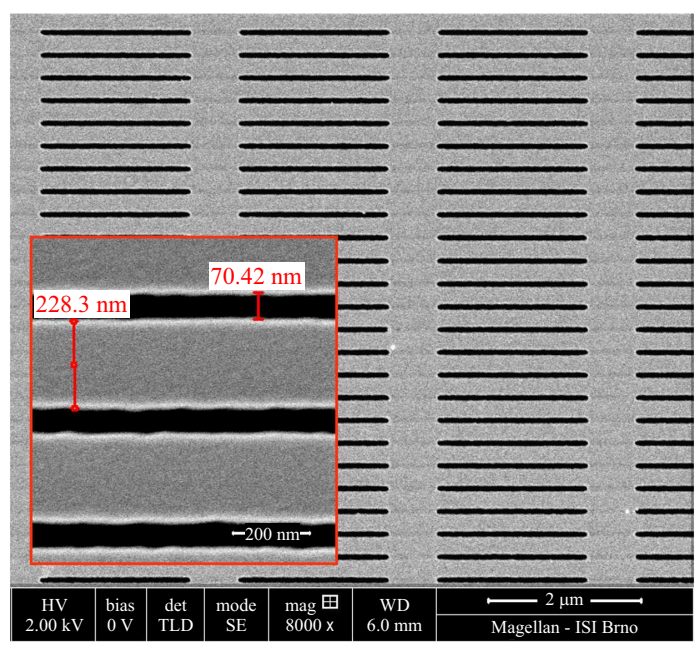

Fig. 5. SEM image of the final holey grating structure fabricated in the thin silicon nitride membrane.size of the rectangle shape opening in the membrane is $70 \mathrm{~nm} \times 2000 \mathrm{~nm}$

\section{Conclusions}

In our work we have described processes used for functional nano-structuring of nitride based freestanding membranes. We point out that both electron beam lithography and selective etching in a RIE based system are very promising in terms of transfer of sub 100 $\mathrm{nm}$ size functional structure into the membrane. During the preparation of the test sample, we suggested possible ways to fine-tune the etching operation in RIE and we proposed recipes for molybdenum hard mask and silicon nitride etching.

Based on several experiments and on our expertise in lithography, we were able to design a functional technological process for the preparation of nanostructured membranes for various applications. The functionality of the presented fabrication procedure was verified with the preparation of a membrane based NOD that was successfully used in the study of electron beam diffraction in the SEM/TEM [14]. We expect a further development and new promising applications for patterned silicon membranes in SEM/TEM system, eg, phase plates, vortexgenerating structures as well as in other fields as laser, EUV, optical fibers light processing and s engineering. 


\section{Acknowledgements}

The research was partially supported by the Technology Agency of the Czech Republic (project TN01000008). The research infrastructure was funded by the Ministry of Education, Youth and Sports of the Czech Republic and the European Commission (project CZ.1.05/2.1.00/01. 0017), and by the Czech Academy of Sciences (project RVO:68081731).

\section{REFERENCES}

[1] E. F. Krimmel, R. Hezel, U. Nohl and R. Bohrer, "Silicon Nitride Integrated Circuit (IC) Technology", Pebler A., Schröder F.Si Silicon.Si.Silicium.Silicon (System-Nr.15), vol S-i/B/1-5/5/c. Springer, pp. 221-222, 1991.

[2] W. Eaton, and J. Smith, "Micromachined pressure sensors: review and recent developments" Smart Materials and Structures, vol. 6, no.5, pp. 530-539, 1997.

[3] G. Rius, A. Baldi, B. Ziaie, M. Z. Atashbar, Introduction to Micro-/Nanofabrication, B. Bhushan (ed) Springer Handbook of Nanotechnology, Springer, ISBN 978-3-662-54355-9, pp. 231-269, 2017.

[4] S. Franssila, Introduction to microfabrication, second edition, Chichester, West Sussex, UK John Wiley \& Sons, ISBN 0-470 -85105-8, pp. 206-216, 2010.

[5] T. Reisinger, S. Eder, M. Greve, H. Smith, and B. Holst, "Free-standing silicon-nitride zoneplates for neutral-helium microscopy", Microelectronic Engineering, vol. 87, no.5-8, pp. 1011 $-1014,2010$.

[6] J. Verbeeck, H. Tian, and A. Béché, "A new way of producing electron vortex probes for STEM", Ultramicroscopy, vol. 113, pp. 83-87, 2012.

[7] E. Anderson, W. Chao, E. Gullikson, S. Rekawa, N. Andresen, and P. Naulleau, "Silicon nitride zoneplates and packaging for EUV instruments", J. Vac. Sci. Technol. B, vol. 31, no.6, pp 06F606, 2013.

[8] T. Niemi, J. Laukkanen, M. Karjalainen and M.Pessa, "Largearea nanoperforated SiN membranes for optical and mechanical filtering", Microelectronic Engineering, vol. 87, no.5-8, pp. $1620-1622,2010$

[9] D. Lee, H. Song, K. Chung, M, Jung, H and Yoon, "Microfabrication of SiN Membrane Nanosieve Using Anisotropic Reactive Ion Etching (ARIE) with an Ar/CF4 Gas Flow", J. Nanosci. Nanotechnol. vol. 11, no. 5 pp. 4511-4516, 2011.

[10] M. Matějka, S. Krátký, T. Řiháček, V. Kolařík, J. Chlumská and M. Urbánek, "Nanopatterning of silicon nitride membranes", In: NANOCON 2016: 8th International Conference Proceedings, Ostrava: Tanger, ISBN 978-80-87294-68-0, pp. 709-714, 2016.

[11] S. Krátký, V. Kolař́ik, M. Matějka, M. Urbánek, M. Horáček and J. Chlumská, "Comparison of Ultimate Resolution Achieved by E-Beam Writers with Gaussian Beam", NANOCON 2013: 5th International Conference Proceedings, Ostrava TANGER, pp. 392-398, 2013.

[12] B. McMorran, J. Perreault, T. Savas, and A. Cronin, "Diffraction of $0.5 \mathrm{keV}$ electrons from free-standing transmission gratings", Ultramicroscopy, vol. 106, no 4-5, pp. 356-364, 2006.
[13] T. Řiháček, F. Mika, M. Matějka and S. Krátký, Müllerová, "Study of the coherence of the primary beam the low energy scanning electron microscope", MC 2015 Microscopy Conference Proceedings, Göttingen DGE, pp. 611-612, 2015.

14] T. Řiháček, F. Mika, M. Matějka and S. Krátký, Müllerová, "Diffraction in a scanning electron", 16th Recent Trends in Charged Particle Optics and Surface Physics Instrumentation Conference Proceedings, Skalský Dvưr, pp. 56-57, 2016.

Received 19 December 2019

Milan Matějka (PhD) was born in 1982 in Brno, Czech Republic. He received his PhD from Brno University of Technology in Brno in 2016. Currently, he works as a post-doctoral researcher at the Institute of Scientific Instruments of the Czech Academy of Sciences where he deals with the experimental and application research in the field of electron beam lithography (EBL), Micro- and Nano- fabrication technologies. In his research activities, he also deals with the development of micro electromechanical systems (MEMS) and optically variable devices (OVDs).

Stanislav Krátký ( $\mathrm{PhD}$ student) was born in 1987 in Ivancice, Czech Republic. He is currently studying (PhD) at Brno University of Technology in Brno. He works as a postgraduate student at the Institute of Scientific Instruments of the Czech Academy of Science where he mainly deals with electron beam lithography. His research is also focused on diffractive optics and other micro and nanotechnologies such as reactive ion etching, UV lithography and various techniques for thin layers preparation.

Tomáš Řiháček (PhD student) was born in 1986 in Brno, Czech Republic. He studied theoretical physics at Masaryk University, Faculty of Science, where he graduated in 2011. Currently, he is a PhD candidate at the Institute of Scientific Instruments of the Czech Academy of Sciences, where he deals with electron diffraction on nanostructures in the scanning electron microscope. He is chiefly interested in electron vortex beams and their incorporation and application in SEM instruments.

Alexandr Knápek (PhD) was born in 1983 in Brno, Czech Republic. He received his PhD from Brno University of Technology in Brno in 2013. Currently, he works as a researcher at the Institute of Scientific Instruments of the Czech Academy of Sciences where he deals mainly with the experimental research of the new cold field emission emitters. His research interest also includes microscopy inspection techniques, instrumentation, and non-orthogonal planar systems.

Vladimír Kolařík (Assoc Prof) was born in 1966 in Brno, Czech Republic. He received his $\mathrm{PhD}$ in microelectronics from the Institute National Polytechnique de Grenoble (France) in 1994. Currently, he works as a researcher at the Institute of Scientific Instruments of the Czech Academy of Sciences. He is internationally known for his work in the field of electron beam lithography, especially within the field of designing function nanostructures and planar optical devices. 